García-Sánchez, F.A., Mirete, A.B., \& Maquilón; J.J. (2013). Implementación y evaluación de webs didácticas para la docencia universitaria presencial. Revista Electrónica Interuniversitaria de Formación del Profesorado, 16(1), 121132.

DOI: http://dx.doi.org/10.6018/reifop.16.1.179481

\title{
Implementación y evaluación de webs didácticas para la docencia universitaria presencial
}

\author{
Francisco Alberto García Sánchez, Ana Belén Mirete Ruiz, Javier J. Maquilón Sánchez \\ Universidad de Murcia
}

\section{Resumen}

Este trabajo es el resultado de un proyecto de innovación docente que es continuación de otros realizados exitosamente en cursos anteriores. Esta experiencia de innovación educativa, realizada para el desarrollo del Campus Virtual de la Universidad de Murcia, ha consistido en el diseño de Webs didácticas para la docencia, teniendo en cuenta unos criterios didácticos, técnicos y pedagógicos preestablecidos, que ha concluido con la implementación real con asignaturas y la evaluación de las mismas por parte del profesorado y estudiantes.

Los participantes en el proyecto han sido catorce profesores y cuatro estudiantes/colaboradores vinculados a la Facultad de Educación de la Universidad de Murcia. Todos han participado en la implementación de las webs didácticas en diecisiete asignaturas, impartidas a veinte grupos de estudiantes de seis titulaciones diferentes, lo que da un total de seiscientos treinta y nueve estudiantes.

Los resultados se presentan desde la perspectiva del profesorado, que evalúa las webs positivamente, ya que ha fomentado su independencia, autonomía y capacidad de autogestión de la docencia; y desde la perspectiva de los estudiantes, que en términos globales las valoran como muy útiles y válidas para la autorregulación de sus aprendizajes.

\section{Palabras clave}

Recursos web; web didáctica; innovación docente; docencia universitaria; autorregulación de aprendizaje. 


\title{
Implementation and assessment of didactics websites for presential university teaching
}

\begin{abstract}
This paper presents a teaching innovation project which has followed others successfully conducted in the past. This project dealt with the development of the online campus of the University of Murcia, Spain. More specifically, we designed didactic websites bearing in mind preset teaching, technical and pedagogical criteria, and implemented them in the teaching and assessment of different subjects.

Fourteen teachers and four students from the Faculty of Education at the University of Murcia (Spain) collaborated in this study. Didactic websites were used in 17 subjects taught in 21 classes in six undergraduate courses. Overall, 639 students benefited from the use of didactic websites.

Results showed that teachers assessed didactic websites positively because these fostered independence, autonomy and self-management in teaching, while students regarded didactic websites as useful and suitable instruments for self-regulation of their learning processes.
\end{abstract}

\section{Key words}

Online resources; didactic website; teaching innovation; university teaching; self-regulation in learning.

\section{Introducción}

Aparejado al desarrollo del Espacio Europeo de Educación Superior (EEES) surge un nuevo modelo educativo centrado en el aprendizaje de los estudiantes, entendido como evolución natural del modelo tradicional de enseñanza centrada en el profesorado (Hernández Pina, García Sanz \& Maquilón, 2001). Como consecuencia, se requiere que hayan variaciones en las concepciones de todos los implicados en dicho proceso de cambio, sobre todo si tenemos en cuenta que los objetivos que persiguen los estudiantes (con sus aprendizajes) y los que buscan los profesores (con sus enseñanzas) también están cambiando (Bauerová \& Sein-Echaluce, 2007).

Desde el inicio de la implantación de la Convergencia Europea los docentes comprometidos con el cambio buscan en la innovación en el aula la respuesta a las nuevas necesidades competenciales requeridas a sus estudiantes. Surgen, por tanto, metodologías acordes a los nuevos roles de profesores y alumnos que buscan facilitar la transición que ambos deben acometer. El aprendizaje por descubrimiento, los estudios de casos, el aprendizaje basado en problemas (ABP), el aprendizaje orientado a proyectos (AOP), el portafolios, el trabajo cooperativo, etc., se han hecho un hueco como metodologías encaminadas a dar cabida a la recién estrenada situación.

De cara a la implantación de las titulaciones de grado y máster, gran parte de las experiencias piloto de adaptación al crédito ECTS se han sustentando en el uso de las tecnologías de la información y la comunicación (TIC), buscando en ellas un desarrollo eficaz y con garantía de calidad de la actividad académica del alumnado universitario (Marín \& Reche, 2011). Podemos entender, por tanto, que las metodologías enfocadas a capacitar al alumno hacia la autogestión de su aprendizaje se encuentran reforzadas si se apoyan en las posibilidades que las TIC tienen para ofrecer a los procesos educativos.

Una realidad que debe asumir la Universidad es la influencia que ejercen las tecnologías sobre todos los ámbitos sociales. No sólo están impregnando el ocio de los jóvenes, o haciendo más cómoda la vida de los ciudadanos, sino que inundan lo profesional, lo académico, lo doméstico... han supuesto la aparición de una nueva organización socioeconómica, política y también el surgimiento de un nuevo modo de comunicarnos, relacionarnos e incluso de trabajar juntos (Adell, 1997, Coll, 2004, Sánchez, Boix \& Jurado, 2009). Ello hace que la Universidad, como institución educativa 
comprometida con el cambio y el progreso social, sea uno de los centros que debieran prestar especial atención tanto a su gestación y evolución, como hacia la capacitación competencial de sus estudiantes. Por las aulas universitarias pasan los profesionales de mañana, esos profesionales que han de desarrollarse como miembros de pleno derecho en esta nueva civilización digital.

Por ello, el profesor, como mediador del proceso de aprendizaje de sus alumnos, deberá posibilitar los medios que sirvan para interaccionar con las TIC, haciéndolos sentir artífices de su formación, con una información que, al tener la posibilidad de obtenerla de fuentes dispares, les permita acrisolarla y formar una opinión crítica y personal de la misma (Sánchez, Boix \& Jurado, 2009).

La adquisición y desarrollo de competencias digitales o tecnológicas en la sociedad de la información y el conocimiento, se torna un aspecto fundamental si el objetivo que nos guía como docentes es generar aprendizajes que capaciten al discente para su constante evolución, desarrollo y aprendizaje a lo largo de toda su vida. Entendido de esta forma, no se trata sólo de estar alfabetizado digitalmente, sino de ser capaz de reaprender constantemente y de adaptarse a los cambios y tecnologías que van surgiendo y transformándose (Area, Gros \& Marzal, 2008).

En la actualidad son muchos y muy diferentes los recursos y herramientas tecnológicas que el docente tiene a su disposición, pero emplearlas de manera que faciliten el aprendizaje a los estudiantes en una asignatura es más complejo. Obviamente, las TIC per sé no representan ninguna garantía al servicio de la enseñanza y el aprendizaje, sino que las posibilidades didácticas que podemos encontrar en ellas van a depender de aquello que demandemos al alumno y las actividades que tengan que realizar con ellas para resolverlo (Area, 2007, Cabero \& López, 2009). Por ello, es básico que el docente esté capacitado, no sólo para aplicar las tecnologías a la educación, sino también para diseñar nuevos escenarios donde los alumnos puedan aprender a moverse e intervenir con las TIC.

En los últimos años, desde el Departamento de Métodos de Investigación y Diagnóstico en Educación de la Universidad de Murcia, se ha desarrollado una línea de trabajo encaminada al diseño, aplicación y evaluación de Webs didácticas para su uso en la docencia universitaria. Éstas se han diseñado con la pretensión de que supongan un recurso para favorecer la inclusión y delimitación de las competencias y contenidos a adquirir, los itinerarios de aprendizaje a seguir, la secuencia de actividades a realizar con recomendaciones y recursos a utilizar; etc. El proceso de diseño y la estructura de las mismas es un complejo proceso que ha sido descrito en múltiples trabajos (García Sánchez \& Martínez Segura, 2009, García Sánchez, Martínez Juárez \& Martínez Segura, 2008, Martínez Segura, García Sánchez \& Martínez Juárez, 2008). También se ha estudiado la capacidad de estos recursos para motivar e implicar al alumnado en las asignaturas (Cabello, García Sánchez \& Mirete, 2009a, 2009b; Mirete, Cabello \& García Sánchez, 2009).

La utilización de estos recursos supone un esfuerzo para el profesor que tiene que conjugar, en su diseño e implementación, su conocimiento en los contenidos de la asignatura; su dominio en las estrategias pedagógicas más adecuadas para su enseñanza; su habilidad para el manejo de las TIC, aprovechando su versatilidad y flexibilidad; y los requerimientos del EEES que pretenden un estudiante participativo, constructor de su propio aprendizaje y colaborador del aprendizaje de sus compañeros.

Por esto, consideramos necesario apoyar los primeros intentos del profesorado en la implementación de este tipo de recursos, para que éste pueda apreciar cómo el recurso favorece el aprendizaje autónomo y autorregulado del alumno, siendo flexible y compatible con diversas estrategias pedagógicas, contribuyendo a adaptar la docencia a los requisitos del EEES y facilitando el trabajo con grupos numerosos de alumnos. A la vez, consideramos necesario desarrollar herramientas y mecanismos eficaces que nos permitan: a) indagar sobre el verdadero uso que el alumnado hace de estos recursos, b) valorar la utilidad que finalmente encuentran en ellos, tanto profesorado como alumnado, c) comparar con efectividad y contrastar los elementos más eficaces y útiles de estos recursos. Por este motivo nos planteamos los siguientes objetivos específicos:

1. Asegurar el diseño y dar apoyo a la implementación de webs didácticas para su uso como apoyo a la docencia presencial. 
2. Valorar la experiencia de innovación con webs didácticas desde la perspectiva de estudiantes y profesores.

\section{Metodología}

\section{Participantes}

Para este trabajo participaron 14 profesores de la Universidad de Murcia (dos Catedráticos de Universidad, tres Titulares de Universidad, cuatro Contratados Doctores, dos Profesores Ayudantes y tres profesores Asociados).

Se valoraron 17 webs didácticas de 7 titulaciones (Grados de Pedagogía, Educación Infantil, Educación Primaria, Educación Social y Logopedia, Licenciatura de Pedagogía y Diplomatura de Magisterio Educación Especial). Del total de asignaturas con Web didáctica, 12 eran cursadas como Obligatorias por los alumnos y solo 5 de todas estas asignaturas eran Optativas. En cuanto a las 5 asignaturas de los nuevos Grados, 3 eran de Formación Básica y 2 Obligatorias.

Finalmente se contó con la participación de 639 estudiantes, de los cuales el $85.5 \%$ eran mujeres y el 14.5\% hombres. En cuanto a la experiencia previa de este alumnado con las TIC, el 38.3\% declararon ser usuarios habituales con fines de ocio, mientras que el $49.7 \%$ manifestaron ser usuarios de internet con fines educativos. Solo un $7.5 \%$ indicaron ser usuarios avanzados o expertos en TIC. La distribución por titulación lo podemos ver en la Tabla 1.

Tabla 1. Distribución de los participantes por titulación

\begin{tabular}{|l|c|c|} 
Titulación & N & $\%$ \\
\hline Grado de Pedagogía & 61 & 9.5 \\
\hline Grado de Educación Social & 118 & 18.5 \\
\hline Grado de Educación Infantil & 66 & 10.3 \\
\hline Grado de Educación Primaria & 120 & 18.8 \\
\hline Grado de Logopedia & 65 & 10.2 \\
\hline Licenciatura de Pedagogía & 145 & 22.7 \\
\hline Diplomatura de Logopedia & 64 & 10.0 \\
\hline Totales & 639 & 100 \\
\hline
\end{tabular}

\section{Instrumentos}

Los instrumentos empleados para recoger la información fueron diseñados específicamente para esta experiencia de innovación. Se explican brevemente a continuación.

\section{Checklist para la evaluación del diseño de las Webs didácticas}

Para facilitar las tareas de evaluación del desarrollo alcanzado por cada WEB didáctica, se optó por utilizar una lista de comprobación o checklist (García Sánchez et al., 2010). Con ella, se valora el grado de preparación de las Webs didácticas. Esta lista, está compuesta por 37 aspectos que han sido considerados como básicos para encontrar en el diseño de una Web didáctica.

\section{Cuestionario para la evaluación de la experiencia por parte del profesorado}

Consiste en un cuestionario que debe cumplimentar el profesorado participante en la innovación de un modo anónimo. Este cuestionario consta de 27 items a los que se acompaña una escala tipo Likert, siendo 1 el valor más bajo y 4 el más alto. Con estos ítems se valoran aspectos relacionados con la implementación de las webs y su propia percepción del proceso realizado. 


\section{Cuestionario para la evaluación de la experiencia por parte del alumnado}

Con el fin de recoger la valoración que el alumnado hace de las Webs didácticas, que han tenido ocasión de utilizar en sus asignatura, se utilizó un cuestionario que está compuesto por 21 ítems a los que se acompaña una escala de valoración tipo Likert de 4 puntos.

También se ha recabado información desde la plataforma web de cada asignaturas, de tal modo que disponemos de los datos de estadísticas de acceso, tanto a través de los contadores de SUMA (plataforma virtual de la Universidad de Murcia), como de contadores de acceso externos.

\section{Procedimiento}

Para el inicio del proyecto de innovación se realizó una reunión inicial, al comienzo del curso académico. En ese momento quedaron asignadas las directrices del desarrollo de la experiencia, así como las responsabilidades de los participantes en el mismo.

Para agilizar el proceso de trabajo del profesorado se contó con responsables de coordinación y con apoyo técnico para el desarrollo de las Webs.

Las responsabilidades del profesorado pueden sintetizarse en:

a) Implementación, mantenimiento y actualización de la web didáctica de su asignatura/s durante el curso académico.

b) Valoración personal detallada de la experiencia de diseño e implementación de la web desde el punto de vista del docente.

c) Facilitar y gestionar el proceso de aplicación de los cuestionarios de valoración por parte de los estudiantes, en el primer o segundo cuatrimestre, según corresponda.

d) Participación en el análisis e interpretación de los resultados obtenidos.

Finalmente, se llevó a cabo la recogida de los datos de acceso y el análisis de los mismos. Se preparó un informe individual para cada profesor con los resultados de la valoración que los estudiantes habían hecho de su web didáctica, y se comenzó el proceso de difusión de los resultados tras la redacción del informe final.

Aún así, pequeñas incidencias han limitado la disponibilidad de algunos datos, por ejemplo en la asignatura Biopatología de la Edad Escolar no llegó a habilitarse el contador externo de visitas. En otras cuatro asignaturas no se activaron los contadores de SUMA y en dos asignaturas no fue factible aplicar el cuestionario de evaluación de la web por parte del alumnado.

\section{Resultados}

En el primero de los objetivos se planteaba la finalidad de asegurar el diseño y dar apoyo a la implantación de webs didácticas para su uso como soporte a la docencia presencial.

Este objetivo se ha alcanzado plenamente, ya que se han desarrollado 17 webs didácticas, que a su vez, han sido empleadas con 20 grupos diferentes de estudiantes universitarios.

Esta afirmación no quiere decir que todas ellas han tenido el mismo grado de desarrollo e implicación por parte del profesorado, como puede observarse en la tabla 2, pero sí podemos afirmar que todas ellas han conseguido un nivel de diseño mínimo para ser consideradas como funcionales.

En la tabla 2 se incluye el grado de desarrollo alcanzado por cada una de las webs didácticas, medido con la checklist en términos de porcentaje. Estos porcentajes se han calculado sobre el total de ítems evaluados (37) salvo que el profesorado justificara que alguno de los ítems no era aplicable a su asignatura, especialmente en los ítems referidos a "elementos específicos de la asignatura" y a la utilización de determinados instrumentos de evaluación, que han sido consideradas como específicas de cada web. 
Tabla 2. Grado de desarrollo de cada web didáctica

\begin{tabular}{|c|c|}
\hline Asignatura & $\%$ desarrollo \\
\hline Bases Orgánicas y Funcionales de la Educación & 100 \\
\hline Biopatología de los Procesos superiores & 100 \\
\hline Análisis y tratamiento de datos en Investigación Educativa & 100 \\
\hline Diagnóstico pedagógico & 87.5 \\
\hline Biopatología de las Deficiencias & 100 \\
\hline Biopatología de la Edad Escolar & 100 \\
\hline Orientación y Diagnóstico Pedagógico en Educación Especial & 37.5 \\
\hline Diagnóstico Pedagógico y observación en Educación Infantil & 68.2 \\
\hline Investigación y TIC & 100 \\
\hline Aspectos preventivos de la Biopatología de las Deficiencias & 96.1 \\
\hline Métodos de investigación en Educación Social & 100 \\
\hline Biopatología Infantojuvenil y NEEs & 100 \\
\hline Modelo Integral de actuación en Atención Temprana & 100 \\
\hline Calidad servicios - personas discapacidad & 96.7 \\
\hline Logopedias Específicas: Intervención & 50 \\
\hline Sistemas Alternativos de Comunicación & 50 \\
\hline Alteraciones logopédicas en los trastornos del desarrollo & 50 \\
\hline
\end{tabular}

Hemos considerado como criterio de calidad en el diseño de las webs que hayan superado el $75 \%$ en su desarrollo. Como se observa en la tabla 2, sólo una asignatura no ha superado el 50\%, se trata de la asignatura orientación y diagnóstico pedagógico en educación especial con un 37.5\%. Otras cuatro asignaturas se encuentran por debajo de este valor, aunque igualan o superan el $50 \%$, se trata de diagnóstico pedagógico y observación en educación infantil con el 68.2\%, logopedias específicas: intervención, con el 50\%, sistemas alternativos de comunicación, con el 50\% y alteraciones logopédicas en los trastornos del desarrollo, también con el $50 \%$.

En el segundo de los objetivos se valora la experiencia de innovación con webs didácticas desde la perspectiva de estudiantes y profesores. En la tabla 3 se recogen las puntuaciones medias obtenidas por cada ítem del cuestionario que ha cumplimentado el profesorado. Recordamos que cada ítem se valora con una escala de 1 (Nada de acuerdo) a 4 (Totalmente de acuerdo).

Como se aprecia en la tabla 3, la valoración general de la experiencia es muy positiva. De hecho, solo tres ítems obtienen puntuaciones por debajo de 3 puntos sobre 4 que son:

Ítem 15 ( $M=2.22$ puntos), sobre la utilidad de la web didáctica para potenciar el trabajo en equipo. Como interpretación de este resultado señalaríamos que, como es obvio, la web didáctica, en si misma, no favorece el trabajo equipo, salvo en el caso puntual de que se hayan diseñado actividades específicas con esa finalidad en la asignatura y en las que la utilización de la web didáctica sea la clave determinante. De no ser así, la web didáctica está pensada más como un recurso para facilitar y orientar el trabajo individual del alumno.

Ítem 19 ( $M=2.56$ puntos), sobre la utilidad de la web didáctica para facilitar y reducir el trabajo de profesores y alumnos. Sería interesante recabar información sobre este ítem en futuros cursos académicos, ya que algunas de las webs se implementaban por primera vez y el volumen de trabajo era percibido por el profesor como que "no compensaba el esfuerzo de diseño de la web con la reducción del trabajo con los estudiantes". 
Tabla 3. Valoración media de los ítems del cuestionario a profesores

\begin{tabular}{|c|c|}
\hline & Media \\
\hline 1. Mi formación en TIC ha sido suficiente para realizar la Web-Home, ponerla en marcha y mantenerla. & 3.0 \\
\hline 2. El empleo de las TIC ha contribuido a la elaboración de materiales útiles para mi asignatura. & 3.78 \\
\hline $\begin{array}{l}\text { 3. Tras utilizarla, veo en la WEB home de la asignatura un recurso para facilitar información al } \\
\text { alumnado. }\end{array}$ & 3.67 \\
\hline $\begin{array}{l}\text { 4. Tras utilizarla, veo en la WEB home de la asignatura un recurso como guía tutorial del aprendizaje } \\
\text { del alumno. }\end{array}$ & 3.33 \\
\hline 5. Tras utilizarla, veo en la WEB home de la asignatura un recurso didáctico. & 3.78 \\
\hline 6. Tras utilizar la WEB home de la asignatura ha aumentado mi interés por ella y sus posibilidades. & 3.33 \\
\hline 7. Los recursos disponibles han sido suficientes y adecuados para la realización de la innovación. & 3.44 \\
\hline 8. He tenido el apoyo que he necesitado en el momento que lo he solicitado. & 3.67 \\
\hline 9. Estoy satisfecho/a con el rol que ha desempeñado el coordinador del proyecto. & 3.89 \\
\hline $\begin{array}{l}\text { 10. La utilización de la WEB-home ha ayudado a los estudiantes a adquirir las competencias } \\
\text { específicas de la asignatura. }\end{array}$ & 3.11 \\
\hline $\begin{array}{l}\text { 11. El empleo de la WEB-home ha facilitado a los estudiantes el desarrollo de las competencias } \\
\text { genéricas o transversales de la asignatura. }\end{array}$ & 3.22 \\
\hline $\begin{array}{l}\text { 12. La incorporación de la WEB-home ha ayudado a los alumnos a asimilar los contenidos de la } \\
\text { disciplina. }\end{array}$ & 3.11 \\
\hline $\begin{array}{l}\text { 13. La utilización de la WEB-home ha fomentado en los alumnos el interés y la motivación hacia la } \\
\text { asignatura. }\end{array}$ & 3.33 \\
\hline 14. El empleo de la WEB-home ha favorecido la interacción profesor-alumno. & 2.87 \\
\hline 15. La incorporación de la WEB-home ha potenciado el trabajo en equipo del alumnado. & 2.22 \\
\hline 16. El empleo de la WEB-home ha facilitado el seguimiento de las clases por los alumnos. & 3.67 \\
\hline 17. La experiencia con la WEB-home ha facilitado el trabajo autónomo de los estudiantes. & 3.89 \\
\hline 18. La introducción de la WEB-home ha propiciado la mejora de la calidad de los aprendizajes. & 3.11 \\
\hline 19. La innovación con la WEB-home ha facilitado y reducido el trabajo de profesores y alumnos. & 2.56 \\
\hline 20. La participación del alumnado en la experiencia ha sido satisfactoria. & 3.67 \\
\hline 21. Los apoyos recibidos de los becarios me han ayudado para el desarrollo de la experiencia. & 3.56 \\
\hline 22. Estoy satisfecho/a con el resultado obtenido para mi asignatura. & 3.44 \\
\hline 23. Mi implicación en el proyecto ha sido adecuada. & 3.33 \\
\hline 24. Considero que este proyecto ha sido de gran utilidad para mi desarrollo profesional. & 3.33 \\
\hline 25. Estaría dispuesto/a a participar en otro proyecto de semejantes características. & 3.56 \\
\hline $\begin{array}{l}\text { 26. Mi concepción sobre la utilidad y uso de las TIC ha mejorado tras la realización de esta } \\
\text { experiencia. }\end{array}$ & 3.0 \\
\hline 27. Seguiré utilizando la Web-Home diseñada dura & 3.78 \\
\hline
\end{tabular}

Ítem $14(M=2.87$ puntos), sobre la utilidad de la web didáctica para favorecer la interacción entre profesores y alumnos. De nuevo, hay que entender que la web didáctica, en si misma, no es un instrumento de interacción bidireccional, como puede ser un chat o foro de discusión. De cualquier modo, la capacidad de estos recursos para favorecer una mayor o menor interacción entre profesor y alumno, no está tanto en el recurso en sí mismo, como en el uso que se haga del mismo.

El cuestionario aplicado al profesorado recoge tres ítems abiertos sobre los puntos fuertes y las propuestas de mejora de la innovación realizada. Tras el análisis cualitativo de los mismos resaltamos que como puntos fuertes se considera que la Web didáctica: 
a) Facilita la información completa de la asignatura a los alumnos desde el principio y de forma concentrada (frecuencia $=4$ ).

b) Facilita la organización de la asignatura (frecuencia $=3$ ).

c) Facilita el trabajo autónomo del estudiante (frecuencia =3).

d) Variedad de webs didácticas creadas e implementadas, generalizarlas a un grupo amplio de profesores (frecuencia $=3$ ).

e) Permite presentar la guía docente de forma más amplia, atractiva y dinámica (frecuencia = 2).

f) La elevada implicación del coordinador y de los becarios del proyecto (frecuencia $=1$ ).

Como aspectos mejorables, se apunta que:

a) Se debe potenciar la sencillez en la elaboración de las webs (frecuencia =6).

b) Se debería compartir el uso que cada profesor hace de la web (frecuencia = 2).

c) Se ha de formar al profesorado en el desarrollo de herramientas web (frecuencia = 1).

En la segunda parte de este objetivo se presenta la valoración de la experiencia por parte del alumnado implicado.

En la tabla 4 se incluye la valoración media de los ítems empleados para que los estudiantes valorasen las webs didácticas de cada asignatura. Se observa que las puntuaciones medias más altas corresponden a los ítems 14 y 20. Esto nos indica que los estudiantes valoran, especialmente alto, el que la web didáctica les permita tener acceso a toda la información de la asignatura desde el comienzo de la asignatura y que estén informados sobre los procedimientos de evaluación de la asignatura.

Tabla 4. Valoración media de los ítems del cuestionario a estudiantes

\begin{tabular}{|c|c|}
\hline Ítem & Media \\
\hline 1.- La web didáctica me ha resultado útil para preparar el examen. & 3.05 \\
\hline 2.- La web didáctica es útil para desarrollar mis aprendizajes. & 3.13 \\
\hline $\begin{array}{l}\text { 3.- La utilización de esta web didáctica ha desarrollado en mi un mayor uso de las tecnologías a } \\
\text { nivel personal/profesional. }\end{array}$ & 2.73 \\
\hline 4.- El tiempo que requiere el uso de la web didáctica me parece adecuado. & 3.06 \\
\hline 5.- Navegar por la página web me ha resultado fácil. & 3.34 \\
\hline 6.- Cuando he entrado en la web didáctica he encontrado lo que buscaba. & 3.23 \\
\hline $\begin{array}{l}\text { 7.- Me ha supuesto un problema el uso de la web didáctica por mis limitaciones de acceso a } \\
\text { Internet }\end{array}$ & 1.69 \\
\hline $\begin{array}{l}\text { 8.- Un obstáculo para trabajar las asignaturas a través de la web didáctica es la falta de formación } \\
\text { que tenemos en el uso de estos recursos. }\end{array}$ & 2.20 \\
\hline 9.- Para poder utilizar la web didáctica no se requiere ninguna formación específica. & 2.88 \\
\hline 10.- Mi uso de la web didáctica ha mejorado con la práctica. & 3.29 \\
\hline 11.- La formación inicial ofrecida por el profesorado para el manejo de la web ha sido útil. & 3.14 \\
\hline 12.- Me gusta cómo está organizada la web didáctica. & 3.19 \\
\hline $\begin{array}{l}\text { 13.- Me gusta que la web didáctica me permita poder trabajar un mismo contenido con diferentes } \\
\text { materiales (texto, power point, imágenes) }\end{array}$ & $3 \cdot 37$ \\
\hline $\begin{array}{l}\text { 14.- La web didáctica debe tener disponible desde el inicio de la asignatura toda la información que } \\
\text { se precisa }\end{array}$ & 3.53 \\
\hline 15.- La web didáctica me ha permitido tener más autonomía en el control de mi aprendizaje & 3.08 \\
\hline 16.- Contar con un cronograma en la web didáctica me ha ayudado a organizar mejor su estudio & 3.00 \\
\hline 17.- Me gusta que la web me haya ofrecido actividades para desarrollar mi aprendizaje & 3.09 \\
\hline $\begin{array}{l}\text { 18.- Me gusta que la web didáctica me haya ofrecido recursos gráficos (imágenes, vídeos, } \\
\text { esquemas...) }\end{array}$ & $3 \cdot 31$ \\
\hline
\end{tabular}




\begin{tabular}{|l|l|}
\hline $\begin{array}{l}\text { 19.- Me gusta que la web didáctica me haya ofrecido información complementaria para ampliar el } \\
\text { contenido (enlaces, artículos...) }\end{array}$ & 3.24 \\
\hline $\begin{array}{l}\text { 20.- Me gusta que la web didáctica me haya ofrecido información sobre cómo se va a evaluar la } \\
\text { asignatura. }\end{array}$ & 3.47 \\
\hline 21.- Valora de forma general tu grado de satisfacción con el uso de la web didáctica. & 3.35 \\
\hline
\end{tabular}

Otras valoraciones muy positivas están corresponden al ítem 13, que apunta que la web permite trabajar un mismo contenido con diferentes materiales. El ítem 18, la web ofrece recursos gráficos. El ítem 5 sobre la facilidad de navegar por la página web y, finalmente, el ítem 21 sobre el grado de satisfacción con la web didáctica.

Finalmente, los ítems con valoraciones más bajas son el 7 y el 8, pero deben ser interpretadas positivamente ya que los mismos estaban formulados en negativo. Es decir, que según estos resultados, los estudiantes han tenido una escasa dificultad para navegar por la web didáctica, ni por limitaciones de acceso a internet, ni por falta de formación por parte de los propios usuarios. También consideramos relevante los datos aportados por el ítem 9 que ha obtenido una valoración media de 2.88 sobre 4 , lo cual indica que para poder utilizar la web didáctica no se requiere ninguna formación específica, haciendo así este recurso accesible para todos los estudiantes independientemente del sexo, edad, nivel económico, curso, titulación o grado de experiencia en el uso de TIC.

\section{Discusión y conclusiones}

Tras la evaluación del diseño y la implementación de las Webs didácticas desde la perspectiva de profesores y estudiantes, disponemos de un conjunto de datos y vivencias que, adecuadamente interpretadas y compartidas en los foros pertinentes, contribuirán a la divulgación de las bondades de las herramientas diseñadas. Es de destacar que todos los datos recogidos apuntan a la valoración altamente positiva de la web didáctica y de sus posibilidades, tanto por alumnos como por profesores.

También se han encontrado hallazgos no previstos (serendipty) ya que se ha conseguido fomentar entre el profesorado la capacidad de adaptar, diseñar y utilizar una herramienta informática que puede estar estrechamente ligada a los requerimientos pedagógicos de la enseñanza de sus propias asignaturas.

Se ha diseñado, solicitando del profesorado una adecuada selección de los contenidos mínimos de sus asignaturas, la estructura básica de una página web con finalidades docentes, que puede ser revisada y actualizada con facilidad por algunas herramientas de diseño de entornos webs.

Podemos afirmar que la finalidad del proyecto se ha cumplido ampliamente ya que consideramos que se han aunado en las webs didácticas, el recurso tecnológico (conocimiento técnico), la capacidad docente y didáctica del profesorado (conocimiento pedagógico) y la aplicación de las mejores estratégicas de enseñanza para cada asignatura (conocimiento del contenido), todo ello en la línea de propuestas como las del Tecnological Pedagogical Content Knowledge (Shulman, 1986, 1987, Mishra y Koehler, 2006). Consiguiendo así, no solo utilizar las TIC, sino hacer un adecuado uso de ellas para nuestros fines específicos (Unwin, 2007).

También valoramos como un resultado derivado de este trabajo el diseño de un instrumento de valoración de recursos web, que permite disponer de un conocimiento detallado y preciso de cuáles son los contenidos incluidos en cada web didáctica, se trata del CEETP, cuyas siglas corresponden a Cuestionario para la Evaluación de Aspectos Didácticos, Técnicos y Pedagógicos de las Webs didácticas (Mirete, Cabello, Martínez \& García Sánchez, 2013).

Las limitaciones de esta investigación han surgido a raíz de los problemas para la implementación completa de alguna web didáctica, para los que habrá que encontrar las soluciones oportunas. 
Por otro lado, como propuesta de mejora, consideramos que se deben articular mecanismos para que el profesorado tenga ocasión de compartir las experiencias acumuladas, que discutan sobre las dinámicas de uso de las webs que cada cual ha empleado y que valoren los resultados obtenidos, tanto desde su propia perspectiva, como desde la de los estudiantes. Opinamos que de ese modo se enriquecería la experiencia profesional, permitiría expandir nuevas ideas y repercutiría en mejoras tangibles sobre la capacidad didáctica de las herramientas docentes con diseño web tienen.

Hemos comprobado como algunas webs incluyen elementos incompletos o bastante mejorables, haciendo de este trabajo algo inconcluso y con proyección de futuro, ya que hemos demostrado que es una herramienta avalada por dos de los grandes pilares de la calidad en la educación (profesores y estudiantes) y, por la existencia de trabajos en los que se establece una relación directa entre el uso de las webs didácticas y el rendimiento académico de los estudiantes (Mirete \& García Sánchez, en prensa), hacen que la prospectiva de futuro de esta línea de investigación sea enorme.

\section{Bibliografía}

Adell, J. (1997). Tendencias en educación en la sociedad de las tecnologías de la información. Edutec, Revista Electrónica de Tecnología Educativa. 7. Disponible en: http://www.uib.es/depart/gte/edutec-e/revelec7/revelec7.html

Area, M. (2007). Algunos principios para el desarrollo de buenas prácticas pedagógicas con las TICS en el aula. Comunicación y pedagogía: Nuevas tecnologías y recursos didácticos, 222, 42-47.

Area, M., Gros, B. \& Marzal, M.A. (2008). Alfabetizaciones y tecnologías de la información y la comunicación. Madrid: Editorial Síntesis.

Bauerová, D. \& Sein-Echaluce, M.L. (2007). Herramientas y metodologías para el trabajo cooperativo en red en la Universidad. Revista Interuniversitaria de Formación del Profesorado, 21 (1), 69-83.

Cabero, J. \& López, E. (2009). Evaluación de materiales multimedia en red en el Espacio Europeo de Educación Superior (EEES). Barcelona: Davinci.

Coll, C (2004). Psicología de la educación y prácticas educativas mediadas por las tecnologías de la información y la comunicación: una mirada constructivista. Sinéctica, 25, pp. 1-24.

García Sánchez, F.A. \& Martínez-Segura, M.J. (2009). Web-docente y aprendizaje: una experiencia en el contexto de la convergencia al EEES. En R. Roig Vila (Dir.). Investigar desde un contexto educativo innovador (pp. 201-217). Alcoy: Marfil.

García Sánchez, F.A., Martínez Segura, M.J., Mirete Ruiz, A. \& Martínez Juárez, M. (2010). Diseño de Webs-Home para asignaturas del Departamento de Métodos de Investigación y Diagnóstico en Educación. En P. Arnaiz, L. Hernández Abenza \& M.P. García Sanz (Eds.). Experiencias de Innovación en la Universidad de Murcia durante 2009 (pp. 115-141). Murcia: Editum.

García Sánchez, F.A., Martínez Juárez, M. \& Martínez Segura, M.J. (2008). Concepto de Web-Home para asignaturas universitarias. En P. Arnaiz, M.P. García Sanz y I. Hernández Abenza (Coords.). III Jornadas sobre el Espacio Europeo de Educación Superior. Murcia: EDITUM. Disponible en línea en http://www.um.es/convergencia/wpcontent/uploads/2008/05/francisco-alberto-garcia.pdf.

Hernández Pina, F., García Sanz, M.P. \& Maquilón, J.J. (2001). Estudio empírico de los enfoques de aprendizaje de los estudiantes universitarios en función del perfil de su titulación (profundo vs superficial). Revista Española de Orientación y Psicopedagogía, 12 (22), 303-318.

Marín, V. \& Reche, E. (2011). La alfabetización digital del alumnado que accede a la Universidad de Córdoba. Edutec-e. Revista Electrónica de Tecnología Educativa, 35. Disponible en: http://edutec.rediris.es/relevec2/relevec35

Martínez Segura, M.J., García Sánchez, F.A. \& Martínez Juárez, M. (2008). Una WEB-Home como herramienta de enseñanza/aprendizaje: una experiencia en el contexto de la convergencia al EEES. [En línea] Comunicación presentada a las VI Jornadas de Redes de Investigación en 
Docencia Universitaria. Vicerrectorado de Planificación Estratégica y Calidad e Instituto de Ciencias de la Educación de la Universidad de Alicante. Alicante, 9 y 10 de Junio. Disponible en http://www.eduonline.ua.es/jornadas2008/comunicaciones/3B7.pdf.

Mirete, A.B., Cabello, F., Martínez Segura, M.J. \& García Sánchez, F.A. (2013). Cuestionario para la Evaluación de aspectos Didácticos, Técnicos y Pedagógicos de Webs Didácticas (CEETP). Murcia: EDITUM. Disponible online en http://libros.um.es/editum/catalog/book/201

Mirete, A.B., Cabello, F., Martínez Segura, M.J., \& García Sánchez, F.A. (2009). Resultados de la aplicación piloto de un instrumento para la evaluación de webs didácticas en asignaturas universitarias. EDITUM: Universidad de Murcia. Disponible en: http://hdl.handle.net/10201/7643

Mirete, A.B. \& García Sánchez, F.A. (Aceptado para su publicación). Rendimiento académico y TIC. Una experiencia con Webs Didácticas en la Universidad de Murcia. Pixel-Bit. Revista de Medios y Educación.

Mishra, P. y Koehler, M.J. (2006). Technological Pedagogical Content Knowledge: A Framework for Teacher Knowledge. Teachers College Record, 108 (6), 1017-1054.

Sánchez Asín, A., Boix, J.L. \& Jurado de los Santos, P. (2009). La Sociedad del Conocimiento y las TICs: una inmejorable oportunidad para el cambio docente. Pixel-Bit. Revista de Medios y Educación, 34, pp.179-204.

Shulman, L.S. (1986). Those who understand: Knowledge growth in teaching. Educational Researcher, $15(2), 4-14$.

Shulman, L. S. (1987). Knowledge and teaching: Foundations of the new reform. Harvard Educational Review, 57 (1), 1-22.

Unwin, A. (2007). The professionalism of the higher education teacher: what's ICT got to do with is? Teaching in Higher Education, 12 (3), 295-308. 


\section{Autores}

Francisco Alberto García Sánchez

Es Doctor en Filosofía y Ciencias de la Educación, Sección de Psicología, por la Universidad de Murcia, Junio de 1989. Catedrático de Universidad del Dpto. de Métodos de Investigación y Diagnóstico en Educación de la Universidad de Murcia. Coordinador del Grupo de Investigación en Educación, Diversidad y Calidad de la Universidad de Murcia (www.um.es/qdiversidad) y del Departamento de Documentación, Investigación y Desarrollo de la Asociación para el Tratamiento del niño con Parálisis Cerebral (ASTRAPACE) de Murcia. Junto a su línea de investigación sobre Atención Temprana y Discapacidad, durante los últimos 10 años viene liderando proyectos de innovación educativa con el uso de webs didácticas y otras TIC en enseñanza universitaria y otros niveles educativos, con una veintena de publicaciones sobre la temática entre artículos y capítulos de libro

Ana Belén Mirete Ruiz

Es Licenciada en Pedagogía y Máster en Psicología de la Educación por la Universidad de Murcia, es becaria de Formación de Profesorado Universitario del programa nacional de recursos humanos de investigación dentro del Plan Nacional de Investigación Científica, Desarrollo e Innovación Tecnológica 2008-2011. Miembro del grupo de investigación E07408 Educación, Diversidad y Calidad, sus líneas de investigación están orientadas hacia la innovación educativa, la incorporación de web didácticas como herramientas mejora de la calidad del aprendizaje y la docencia universitaria. Ha participado en varios proyectos de investigación e innovación educativa, y tiene diferentes publicaciones sobre estas y otras temáticas

\section{Javier J. Maquilón Sánchez}

Es Profesor Contratado Doctor del Departamento de Métodos de Investigación y Diagnóstico en Educación de la Universidad de Murcia. Sus principales líneas de investigación se centran en la identificación de los enfoques de enseñanza y aprendizaje en los niveles de educación primaria, secundaria y universidad, además de la evaluación de los aprendizajes de los estudiantes, del profesorado y de los programas de desarrollo de las habilidades en el estudio. Ha participado y coordinado diversos proyectos de investigación e innovación educativa orientados al EEES, impartido ponencias, cursos de formación de profesorado, presentado comunicaciones y publicado numerosos trabajos entre artículos, libros y capítulos de libro sobre ésta y otras temáticas 